\title{
The logical closure limit of superconductivity descriptions
}

\author{
Z. Bousnane ${ }^{1}$, N. Merabtine' ${ }^{2}$, M. Benslama ${ }^{2}$, F. Boussaad ${ }^{1}$ \\ ${ }^{1}$ Physics Department, Faculty of Science, University of Batna, 05000, Algeria \\ ${ }^{2}$ Electromagnetism and Telecommunication Laboratory, Electronics Department \\ Faculty of Engineering, University of Constantine, 25000 Algeria \\ E-mail:na_merabtine@hotmail.com,malekbenslama@hotmail.com
}

\begin{abstract}
The quantum description of macroscopic behaviour seems requiring the existence of limits imposed by the consideration hold on the logical closure of the theory, according to this, the J.A. Wheeler black box [1] will act under a coherence length of second order. The use of mathematical beings translating the phases transitions in nature are displayed in one-way, the reversibility parameter associated to the black box is severely limited according to the way we disturb the constants and the functions used to describe a given phenomenon. The combinatory formulation of matter is always accompanied by the length scales orientation phenomenon that must be seen as a measurement process of second order. The non-reproducibility of experiments will be dealing with the non-account of the amplitude of length scales interactions, this amplitude manages the combinatory of macroscopic levels between an observer and observed. Some introduced concepts as an entropic potential, the breaking of equivalence are making the emergence of picture of superconductivity as reproducible phenomenon according to the "intrinsic states reproducibility" with number estimated on universal constants expressions limited by the difference between cooled and cooling.
\end{abstract}

Keywords: superconductivity, logical closure limit, equivalence breaking, entropic potential, dodecapolar Riemann's sphere, length scale interaction.

Manuscript received 21.02.06; accepted for publication 23.10.06.

\section{Introduction}

Since its appearance, the superconductivity phenomenon was an external result against the logical coherence of quantum theory considered at this time. It's simple expression as a second variation of classical being mentioned a state of reversibility caused by a multivocity of principles, because the second variation was expressing the direction of variation of physical quantities.

\section{Considerations on the phenomenological pattern of Ginzburg-Landau}

Which was natural extrapolation of quantum description by the fact that quantum is quantum, in a such way that propagation of superconductivity is causally linked to the current density. The causality principles against this must be limited by the non-reversibility of the process, which will be expressed by an equivalence between series development of physical quantities.

\section{The breaking of equivalence}

The interaction concept in the L. Landau description [2] was reformulating parametrically the results as supposing that, during the propagation at every point $T$, the partial equilibrium was considered as a perfect equilibrium with regard to the causality between current density and order parameter, also by assuming a coherence length propagation that fixed the limits of spatial extension and the orientation of order parameter, the microscopic pattern hold on the validity of causality, when order parameter is locally measured, introduced the pairing process by coupling, to preserve for ever quantum for quantum.

The concept quantum without quantum will seriously break the equivalence of the two-ways propagating of phenomenon, the time flowing felt during the first propagation will be not the same by the next, this permit the existence of macroscopic levels.

The introduction of recursive picture of processes can answer the quantum without quantum by 
considering at the same level with regard to the effect of a body and the vacuum surrounded him, this vacuum must be realized by the reflected length coherence.

The universality of indetermination order making the quantum for quantum and also making the equivalence will obey to a jumps rule.

\section{Variational character}

As used among the whole models was universal, by introducing the concept of semi-extremums, the phenomenon of propagation of superconductivity will be shared between the causality of its appearance and causality of its stability, the linear combination of these two causalities must be regarded as giving the stability of the state as the cohabitation of natural indetermination orders, which mathematically are the multicommutation relations limited by a functions of universal constants.

The interference of thermodynamical length scales of temperatures itself is equivalent to a measurement process causing the first order logical opening of the theory, this measurement process gives a quantities corresponding to spins of fluctuating order parameter, this is expressed as internal and external characterizations of magnetic properties.

\section{Dodecapolar Riemann's sphere}

This mathematical being is introduced to give "an optical feature of the stereographic projection", the normalized features are chosen in such a way that the stereographic projection must be regulated by the Descartes relation. This leads to a non-locality of the complex numbers and functions as an order parameter.

The twelve nodes as intersections of the principle circles are representing the intrinsic indetermination orders, which are quantum without quantum.

The Young diagrams corresponding to each node are characterized by a degree of recursivity proportional to the indetermination order at this node.

\section{Entropic potential concept}

Is introduced to include a thermal reversion when the reduced action hold on the statistical weights will take an extremal value. This also obliges to consider the "radial natural elements", this is explained by the fact that the correlation of different natural elements for a given length coherence that is a functional of mean free path, this cohabitation is causing vacuum having the same physical effects as elements with atomic numbers upper to 104.
The representation of the radial natural elements will be given by the solution of the complete Schroedinger equation on $E_{0}^{\sqrt{2}} E_{0}^{\sqrt{2}} E_{0}^{\sqrt{2}} E_{0}^{\sqrt{2}}$ manifold.

\section{The interaction cooled-cooling}

Gives the evidence of effective indetermination related to the macroscopic character of temperature [3] by considering the entropy as the amplitude of the entropic potential manifold curvature under the effect of entropy oscillations, the lateness of reduced statistical action will imply the universality of macroscopic character limited by Boltzmann's constant.

The study of the interaction cooling-cooled will be expressed by the ratio between two temperatures length scales, if this ratio is physically equivalent to a "symmetry propagation" length scale - by inducing recursively a symmetry with different order - the decrease of temperature will associate to every point in the interval $T_{C}-T_{c}$, the unity of action (if the temperature of cooling $T_{C}$ is mentioned by action unites, the cooled will be appreciated as a fluctuation of the cooling temperature).

\section{Conclusion}

To translate a phenomenological meaning based on the existence of macroscopic levels, our essay is rooted in the fact that the combinatory formulation of matter is always accompanied by the length scales orientation phenomenon that must be seen as a measurement process of second order, in other words, we shall say that the combinatory length scales of physical quantities will be a measurement process that have transited at the second order.

The non-reproducibility of experiments will be dealing with the non-account of the amplitude of length scales interaction, because this amplitude manages the combinatory of macroscopic levels between the observer and observed.

\section{References}

1. C.W. Misner, K.S. Thorne and J.A. Wheeler, Inspired from vacuum fluctuation. "Gravitation" Company, San Fransisco, 1973.

2. L. Landau, E. Lifshits, Quantum mechanics. Edition MIR, Moscow, 1967.

3. L. Landau, E. Lifshits, Statistical physics. Edition MIR, Moscow, 1967. 